\title{
Conocimiento etnobotánico de las plantas utilizadas en Chancah Veracruz, Quintana Roo, México
}

José Guadalupe Chan-Quijano* El Colegio de la Frontera Sur Meybi Karina Pat-Canché Jerónimo Saragos-Méndez Universidad de Quintana Roo Unidad Cozumel

\section{Resumen}

Ante la preocupación por conservar la biodiversidad, y dada la creciente necesidad regional por aumentar en la etnobotánica la sustentabilidad de las plantas medicinales, en el poblado de Chancah Veracruz, Quintana Roo, se generó información básica acerca del uso de las plantas de la región. Con base en entrevistas a los habitantes del lugar, se determinó que $53 \%$ de ellos utiliza las plantas con fines medicinales, $20 \%$ para su alimentación, $20 \%$ como medicinas y alimento y $7 \%$ como elementos ornamentales y artesanales. Se identificaron 29 especies aprovechadas por los pobladores, en su mayoría mujeres. Se conservaron tanto la clasificación como los usos de las especies empleadas en el poblado, ya que se observa una relación muy estrecha entre el entorno (plantas) y la población. En este sentido, se cumple la principal característica de la etnobotánica, encargada de estudiar la interrelación entre los seres humanos y las plantas a través del tiempo y de los distintos ambientes culturales y ecológicos.

Palabras clave

Chancah Veracruz, Felipe Carrillo Puerto, Quintana Roo, etnobotánica, especies arbóreas. 


\section{Etnobotanical knowledge of plants used in Chancah Veracruz, Quintana Roo, Mexico}

José Guadalupe Chan-Quijano El Colegio de la Frontera Sur Meybi Karina Pat-Canché Jerónimo Saragos-Méndez Universidad de Quintana Roo Unidad Académica Cozumel

\section{Abstract}

In response to concern for conserving biodiversity and given the growing need for regional increase in ethnobotany sustainability of medicinal plants, in the village of Chancah Veracruz, Quintana Roo, basic information about the use of regional plants was produced. Based on interviews with the inhabitants, it was determined that 53 per cent used plants for medicinal purposes, 20 per cent for their food, 20 per cent as medicines and food, and seven per cent as ornamental items and crafts. We identified 29 species that are used by the villagers - mostly women. Both, the classification and the uses of each species employed in the village were retained as evidence of a very close relationship between the environment (plants) and its inhabitants which fits the main feature of ethnobotany: study the interrelation between human beings and plants through time in different cultural and ecological environments.

\section{KEY WORDS}

Chancah Veracruz, Felipe Carrillo Puerto, Quintana Roo, ethnobotany, tree species. 


\section{Introducción}

Una de las áreas más interesantes del campo interdisciplinario de la etnobotánica es la referente a la percepción, denominación y ordenamiento de las discontinuidades propias del universo vegetal con el que se relacionan los miembros de una determinada comunidad cultural (Barrera, 1979). La cultura botánica tradicional tiene en Quintana Roo una trayectoria única (Chan-Quijano y López-Mejía, 2012: 20), pues los campesinos mayas que habitan en el estado siguen utilizando los recursos vegetales y su conocimiento de ellos; muestra destacada de esto son los programas de manejo forestal con los que logran, de manera eficiente, el adecuado aprovechamiento y la conservación de sus selvas, ya que consideran al monte como su principal capital y no como una inversión especulativa (Galletti, 1999; Anta-Fonseca y Pérez-Delgado, 2006: 61; RebollarDomínguez , 2008). Además, el conocimiento tradicional de los campesinos permite generar información básica acerca del uso de las plantas y motiva la conservación de la biodiversidad, que a su vez estimula la necesidad regional por aumentar -en la etnobotánica- la sustentabilidad de las plantas medicinales. En la actualidad, el uso de los recursos naturales implica el aprovechamiento de todos los ambientes ecológicos existentes, que, en el caso de este ejido, son: selva mediana, vegetación secundaria, sabana, cenotes, aguadas y lagunas, mediante actividades como la milpa, el huerto familiar y el ganado de traspatio en el solar; la recolección de plantas silvestres, la cacería y la pesca, así como el uso forestal comercial (Bello-Baltazar ., 2002). El objetivo de esta investigación es sistematizar el conocimiento etnobotánico de las plantas utilizadas por los pobladores de la comunidad de Chancah Veracruz.

\section{Antecedentes}

La etnobotánica es el campo interdisciplinario que comprende el estudio e interpretación del conocimiento tradicional de elementos de la flora. Por tradicional se entiende que tales conocimientos, con valor cultural, manejo y usos, han sido transmitidos y hechos suyos a través del tiempo por un grupo humano, dentro de un determinado contexto cultural. En este texto, la raíz se concibe como pueblo, pero no en un sentido racial, sino social y cultural. En pocas palabras, el principal objeto de la etnobotánica son las sabidurías botánicas tradicionales (Barrera-Marín, 2012). Desde épocas remotas la sociedad utilizó las plantas con 
fines alimenticios y medicinales, este saber se fue acumulando con el paso del tiempo, pasando de un individuo a otro por tradición oral (Hernández-Álvarez, Ávila-Uribe y Patiño-Siciliano, 2009: 9). En las comunidades carentes de servicios médicos, las parteras, los yerberos y los curanderos son los responsables de la salud de los pobladores, pero la medicina tradicional también es utilizada por los enfermos en lugares donde hay servicios médicos. En las áreas marginadas existen grupos indígenas con grandes conocimientos de la herbolaria medicinal, alimenticia y artesanal, así como del ambiente donde se desarrollan estas plantas. Tales conocimientos, acopiados durante generaciones, constituyen la base de la biodiversidad cultural global y del uso sustentable de estos recursos (Leonti, Sticher y Heinrich, 2003; Canales ., 2006; Gómez-Álvarez, 2012), por lo que es necesario hacer esfuerzos para evitar su pérdida definitiva.

\section{Materiales y métodos}

El ejido de Chancah Veracruz (centro ceremonial maya) cuenta con 325 habitantes y se ubica entre los paralelos $19^{\circ} 30^{\prime}$ y $20^{\circ} 45^{\prime}$ de latitud Norte y en los meridianos $88^{\circ} 45^{\prime}$ y $87^{\circ} 30^{\prime}$ de longitud Oeste, a $25 \mathrm{msnm}$, en el municipio de Felipe Carrillo Puerto, Quintana Roo. Colinda al Norte con el ejido de Felipe Carrillo Puerto, al Oeste con el ejido de Santa Isabel, al Este con la Reserva de la Biósfera de Sian Ka'an y con la comunidad de Uh May (Varela-Carlos, 1998; Rebollar-Domínguez ., 2008).

Se realizaron dos visitas a la comunidad para recolectar datos sobre la diversidad etnobotánica. Se efectuaron 100 entrevistas uniestructuradas con preguntas abiertas, en las cuales el informante pudo comentar libremente el contenido de su respuesta; considerando estas, se recolectaron muestras de las plantas mencionadas en la selva y en los huertos familiares (Olmsted, López-Ornat y Durán-García, 1983; Camus y Bustamante-Araya, 1998: 82; Bautista-Zúniga ., 2004: 506; Rebollar-Domínguez , 2008). Los métodos empleados para el acopio de la comunidad vegetal fueron: la colecta, el prensado, el secado y el montaje; siguiendo las técnicas de Cruz (2000) y Villarreal-Quintanilla (2002: 151).

Los ejemplares botánicos fueron identificados por sus características dendrológicas (morfológicas) y mediante comparación con material del herbario de la Universidad de Quintana Roo, Campus Cozumel, y de las siguientes claves taxonómicas: Bonnier (1990: 326), Ochoa-Gaona (1990: 261; 2012: 299), 
Pulido-Salas y Serralta-Peraza (1993: 106), Bonnier y De Layens (1999: 405), Ramos-Prado y Porter-Bolland (2002: 14), Anderson (2005: 199); Ochoa-Gaona, Pérez-Hernández y Jiménez-Pérez (2008: 137) y Zamora-Cornelio (2010). Las muestras colectadas se encuentran bajo resguardo de la señora Guillermina Noh Guille, habitante de la comunidad, para su revisión por los mismos habitantes y personas que los visiten.

\section{Resultados}

Se registraron 29 especies con uso tradicional (gráfica 1) que pertenecen a 23 familias; $53 \%$ es de uso medicinal, $20 \%$ de autoconsumo (alimento), $20 \%$ para ambos usos y $7 \%$ para fines ornamentales, por ejemplo la elaboración de artesanías, entre otras cosas. Los resultados de este estudio revelan una parte del conocimiento tradicional en el uso de plantas medicinales por los pobladores, y explican parcialmente la contribución de las plantas medicinales a la sustentabilidad.

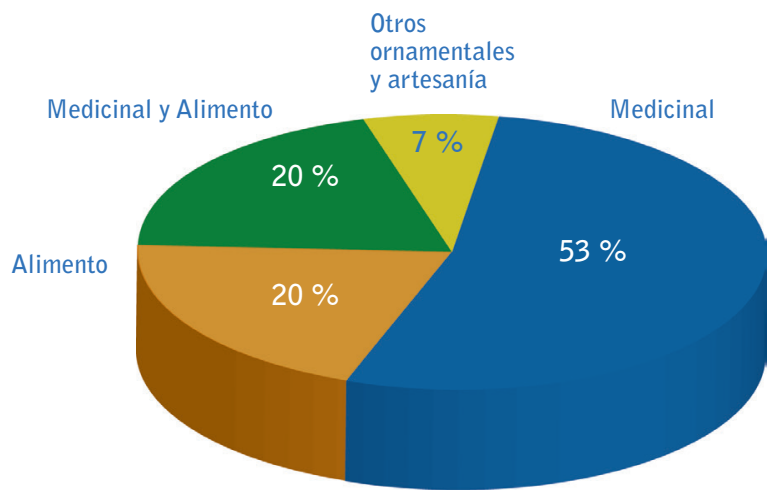

Gráfica 1. Uso de las plantas por los pobaldores de Chancah, Veracruz, Quintana Roo

La utilización de estas especies emprende el desarrollo de la etnobotánica dentro de la comunidad favoreciendo un programa de ventas de artesanías, un mercado de plantas ornamentales, medicinales y comestibles y de jardines demostrativos. Las plantas encontradas y el uso que le dan en la comunidad se muestran en el cuadro 1. 


\section{Cuadro 1. Especies encontradas en Chancah Veracruz, y principales usos por los pobladores}

\begin{tabular}{|c|c|}
\hline ESPECIE & Uso \\
\hline $\begin{array}{l}\text { Nombre científico: } \\
\text { Acrocomia mexicana } \\
\text { Familia: Palmaceae } \\
\text { Nombre común: Cocoyol } \\
\text { Nombre maya: Tuk' }\end{array}$ & $\begin{array}{l}\text { Medicinal: La raíz se usa para la diabetes. Los aceites de la } \\
\text { semilla y la pulpa se emplean en la fabricación de jabones lla- } \\
\text { mados coco. La pulpa es comestible tanto fresca como pren- } \\
\text { sada, también de ella se extrae un aceite ligero y por medio } \\
\text { de la fermentación se obtiene un licor de sabor agradable. La } \\
\text { semilla se puede comer asada o cocida. El interior del tronco } \\
\text { se muele para obtener una harina muy fina y sabrosa, y el } \\
\text { cogollo se consume como palmito. Las hojas se usan como } \\
\text { forraje para el ganado rústico y, tras una cuidada maceración, } \\
\text { se extrae de ellas una fibra útil en la confección de sogas, } \\
\text { redes, etcétera. }\end{array}$ \\
\hline $\begin{array}{l}\text { Nombre científico: } \\
\text { Aloe vera } \\
\text { Familia: Lilianaceas } \\
\text { Nombre común: Sábila } \\
\text { Nombre maya: Yucca }\end{array}$ & $\begin{array}{l}\text { Medicinal: La resina se toma para la digestión, úlceras, estre- } \\
\text { ñimiento, infecciones renales, mal funcionamiento del híga- } \\
\text { do, presión arterial, aumento del apetito, insomnio y dolores } \\
\text { de cabeza. Para el cabello se toma la gelatina antes de las } \\
\text { comidas durante siete días. Para la inflamación de la vesícula } \\
\text { biliar, infecciones renales y disfunciones hepáticas se pone a } \\
\text { remojar una hoja de sábila por } 12 \text { horas para extraer el yodo } \\
\text { que contiene. Se extrae la gelatina de la penca, se lava y se ra- } \\
\text { lla una papa blanca con su cáscara (sin pelarla) y se mezclan } \\
\text { ambos, el jugo de la papa con la gelatina (mitad y mitad), la } \\
\text { gelatina sobrante se guarda para usarse en otro preparado } \\
\text { (no guardar por más de } 12 \text { horas). El preparado se toma en } \\
\text { ayunas. Para las personas o niños que no desean comer: una } \\
\text { cucharada de la gelatina con miel de abeja antes de ir a dor- } \\
\text { mir y otra en ayunas al despertar. }\end{array}$ \\
\hline
\end{tabular}

Nombre científico: Alseis yucatanensis Familia: Rubiaceae Nombre común: Papelillo Nombre maya: Petcacab
Artesanal: Se emplea para la elaboración de durmientes, por su dureza. 


\section{Cuadro 1. Especies encontradas en Chancah Veracruz, y principales usos por los pobladores \\ (continuación)}

ESPECIE

Nombre científico:

Artemisia ludoviciana ssp mexicana

Familia: Asteraceae Nombre común: Zisim

Nombre maya: Zizim

Nombre científico: Bromelia chrysantha Familia: Cactaceae Nombre común: Piñuela Nombre maya: Cadena ch'om

Nombre científico: Brosimum alicastrum Familia: Moraceae Nombre común: Ramón Nombre maya: $O x x$

Nombre científico: Byrsonima crassifolia Familia: Malpighiaceae Nombre común: Nance Nombre maya: Chil

Nombre científico: Carica papaya

Familia: Caricaceae Nombre común: Papaya Nombre maya: Ch'ich'put
Uso

Medicinal: Para vómito: infusión. La planta se hierve y se utiliza para la pleuresía en una aplicación caliente; también para la tos, el asma y la diarrea. Además, para los cólicos se coloca un cataplasma de esta planta directamente en el abdomen.

Medicinal: Para el mal de ojo y la disentería. Su leche se utiliza para las manchas, con mucha cautela porque puede producir quemaduras en la piel.

Medicinal y alimento (animales): Látex, hojas (en infusión): antitusivo, balsámico, diaforético, emenagogo y para enfermedades como asma, diabetes, tisis, tuberculosis, bronquitis. La corteza, en infusión, se usa como tónico. Sirve como alimento para animales (borrego y jabalí).

Alimento y medicinal: La infusión de vapores de la corteza es astringente y se emplea para detener la diarrea y, en algunas ocasiones, para bajar la fiebre; también se utiliza la corteza pulverizada como medicamento para contrarrestar las úlceras.

Alimento (animales): Prima de la papaya, es más chiquita, las hojas se dan a los animales (jabalí) como alimento. 


\section{Cuadro 1. Especies encontradas en Chancah Veracruz, y principales usos por los pobladores \\ (continuación)}

ESPECIE

Nombre científico: Cecropia peltata

Familia: Moraceae Nombre común: Guarumo (hoja tiernita del)

Nombre maya: Xk'o'och

Nombre científico:

Chenopodium ambrosioides

Familia: Chenopodiaceae

Nombre común: Epazote Nombre maya: Kuku'um xiw

\section{Nombre científico:}

Cissampelos pareira

Familia: Menispermaceae Nombre común: Oreja de ratón

Nombre maya: X'petetum

Nombre científico: Citrus aurantium

Familia: Rutaceae

Nombre común: Naranja (hoja de)

Nombre maya: Ch'ujuc

Pa'aal
Uso

Medicinal: Se emplea contra diabetes, asma, mal de San Vito o de Parkinson, padecimientos hepáticos, obesidad y para eliminar verrugas. También aprovechan sus propiedades diuréticas y digitálicas (aumenta la fuerza cardiaca). Se recomienda para mitigar los efectos de la diabetes y las afecciones pulmonares: con las hojas se prepara una infusión y se administra de forma oral. Para curar las heridas se aplica un emplasto elaborado con las hojas. Se prepara sancochado para la disentería.

Medicinal y alimento: Se usa para expulsar parásitos intestinales, sobre todo del tipo áscaris y anquilostomas. Después de utilizarla se debe administrar un laxante fuerte para expulsar los parásitos muertos. Las hojas tienen un efecto antiespasmódico y también contrarrestan las flatulencias. Infusión: 30 gramos por litro de leche caliente, junto con alguna planta aromática. Extracto líquido: de 0.5 a 5 gramos diarios.

Medicinal: Para mal de ojo y disentería, un manojo de hojas de esta planta junto con las del pech'uk' (Porophyllum macrocephalum), sina'anche', tsutsul y ruda; se machacan juntas y se agregan seis litros de agua; con este preparado se baña al niño enfermo.

Medicinal: Para el vómito y para que las mamás tengan más leche, como sedante ligero, antiespasmódico y digestivo. El jarabe de la corteza se usa en el tratamiento contra la fragilidad capilar. El naranjo amargo sirve de porta injertos. Esta especie es más resistente a enfermedades y condiciones de suelo que otros cítricos, de ahí que se haya extendido ampliamente su cultivo como pie para injertar otros cítricos. Muy usado como especie ornamental por la vistosidad de sus flores y por su aroma en alineaciones de naranjo amargo o como ejemplar solitario. 


\section{Cuadro 1. Especies encontradas en Chancah Veracruz, y principales usos por los pobladores \\ (continuación)}

ESPECIE

Nombre científico:

Cnidoscolus chayamansa

Familia: Euforbiácea

Nombre común: Chaya

Nombre maya: Chay

Nombre científico: Cordia sebestena

Familia: Boraginaceae

Nombre común: Coopte

Nombre maya: Chakopte

Nombre científico:

Crescentia cujete

Familia: Bignoniaceae

Nombre común: Jícara

Nombre maya: Luuch

Nombre científico:

Cucurbita pepo

Familia: Cucurbitáceas

Nombre común: Calabaza

(hoja de)

Nombre maya: $X-k a ́$

Nombre científico: Hamelia

patens

Familia: Rubiaceae

Nombre común: Bencenuco

Nombre maya: Xcanan
Uso

Medicinal y alimento: Las hojas frescas licuadas en agua se utilizan para combatir diversos padecimientos: anemia, asma, cáncer, colesterol, diabetes e hipertensión.

Medicinal: Infusión, para la tos se bebe como té.

Artesanía y alimento: Se utiliza en las artesanías, como combustible, alimento (refresco, aceite), forraje (fruto), medicina y como melífero. Una bebida muy nutritiva y apreciada para el consumo humano (horchata) es producto de las semillas de este fruto; su disponibilidad en el mercado es bastante baja debido a la rudimentaria forma de extraer las semillas de la pulpa: macerándolas con suficiente agua, método manual lento y tedioso. También se obtienen los tradicionales y antañones guacales, vasos y cucharas; con su cáscara se construyen juguetes como enchutes y helicópteros. Además de la harina, de la semilla se extrae aceite, que resulta excelente para su uso alimenticio. Con un tratamiento específico se utiliza como sustituto del carburante diesel.

Medicinal: En la milpa para picaduras de serpiente, baja el veneno y da tiempo para ir al doctor.

Medicinal: Se aplica macerado en los piquetes de mosco para mitigar la comezón. 


\section{Cuadro 1. Especies encontradas en Chancah Veracruz, y principales usos por los pobladores \\ (continuación)}

ESPECIE

Nombre científico:

Manilkara zapota

Familia: Sapotaceae

Nombre común:

Chicozapote

Nombre maya: Sak-yá

Nombre científico: Mentha

piperita

Familia: Labiatae

Nombre común:

Yerbabuena

Nombre maya: Xakil xiw

Nombre científico: Morinda citrifolia

Familia: Rubiaceae

Nombre común: Noni

Nombre maya: Oon
Alimento: Fruto comestible. Uno de sus principales productos es el látex, empleado para elaborar chicle. El fruto fresco es muy apreciado y con él se confeccionan mermeladas y jarabes por su agradable sabor dulce. Es objeto de comercio en los mercados regionales.

Medicinal y alimento: Como se trata de una planta con aceite esencial, sus propiedades medicinales son las comunes a todas estas especies: sedante, antiespasmódica, tónica y digestiva. También posee cualidades expectorantes, ya que se absorbe y elimina por las vías respiratorias y las descongestiona. Por estas propiedades, es de gran utilidad en el tratamiento de la patología congestiva: rinitis y bronquitis. El mentol de esta planta posee un efecto refrescante al aplicarse sobre la piel; calma las molestias del prurito y de las quemaduras. Aplicado sobre las sienes, también alivia la cefalea.

Medicinal y alimento: El fruto se usa en el tratamiento de la diabetes, problemas del corazón y presión alta, así como para la tuberculosis, la artritis, el reumatismo, la menstruación dolorosa, las úlceras gástricas, las infecciones e incluso la depresión. Los extractos de la hoja se usan para controlar el flujo excesivo de sangre y evitar la formación de coágulos; estudios recientes comprueban su actividad contra los tumores. Las infusiones de la hoja ayudan en el tratamiento de fiebres y desórdenes en los niveles del azúcar en la sangre, desórdenes urinarios, hinchazón, dolores musculares y de las articulaciones. Las hojas son particularmente valiosas en el tratamiento de condiciones inflamatorias y cicatrización de la piel; pues contienen ácido ursólico, con propiedades anticancerosas, así como B-sitosterol, que puede disminuir de manera importante los niveles de colesterol. También son ricas en vitaminas y minerales y contienen altos niveles de fósforo, hierro, calcio, magnesio, vitamina E, vitamina $\mathrm{K} 1$ y niacina. 


\section{Cuadro 1. Especies encontradas en Chancah Veracruz, y principales usos por los pobladores \\ (continuación)}

ESPECIE

Nombre científico: Musa sapientum

Familia: Musáceas

Nombre común: Plátano

Nombre maya: $H a$ 'as

Nombre científico: Ocimum basilicum

Familia: Labiadas

Nombre común: Albahaca Nombre maya: Hoch ol ka'al

Nombre científico: Opuntia robusta

Familia: Cactaceae

Nombre común: Nopal Nombre maya: Pa'can
Uso

Alimento y medicinal: Las hojas se usan para tamales y los frutos son comestibles. Tiene propiedades benéficas para controlar la diarrea, es astringente, regula el tránsito intestinal, hipertensión, sistema nervioso y muscular, anemia, gota. Refuerza el sistema inmunológico, y ayuda en la formación de anticuerpos, dientes, huesos, así como de glóbulos rojos y blancos. Combate problemas cardiovasculares y previene el cáncer de colon. Está contraindicado si se padece insuficiencia renal.

Medicinal y alimento: Con alcohol, mojando un trapo, para la fiebre y el mal alimento. Favorece la digestión y evita los espasmos gástricos; es muy útil en los casos de gastritis, hernia de hiato, flato, etc. Infusión de un puñado de hojas frescas, unos 15 gramos por litro de agua; tomar tres tazas al día después de las comidas. La esencia de la planta abre el apetito (dos o tres gotas al día disueltas en azúcar). Estimula la producción de leche en las mujeres lactantes (decocción de 30 gramos de hojas secas por litro de agua, dos tazas diarias).

Medicinal: Para la obesidad, pues gracias a su gran cantidad de fibra, ayuda a retardar el tiempo en que se absorben los nutrimentos y entran a la sangre, por lo que facilita su eliminación. Diabetes e hiperglucemia: también se dice que ayuda en el tratamiento de la diabetes. Incrementa los niveles y la sensibilidad a la insulina, con lo que estabiliza y regula el nivel de azúcar en la sangre. En personas con colesterol elevado se ha demostrado que su consumo ayuda a eliminarlo, evitando que se absorba gran parte de este y se acumule en venas y arterias. Los aminoácidos, la fibra y la niacina contenida en el nopal previenen que el exceso de azúcar en la sangre se convierta en grasa, además de que actúa metabolizando la grasa y los ácidos grasos, reduciendo el colesterol. Antibiótico, previene el cáncer, los desórdenes gastrointestinales y mejora la digestión. 


\section{Cuadro 1. Especies encontradas en Chancah Veracruz, y principales usos por los pobladores \\ (continuación)}

ESPECIE

Nombre científico:

Origanum vulgare

Familia: Labiatae

Nombre común: Orégano grande (hoja de)

Nombre maya: Ak'il ché

Nombre científico: Piper

auritum

Familia: Piperaceae

Nombre común: Momo

Nombre maya: Mak'olam o

X-mak-ulam

Planta no identificada

Nombre común: Quitanche

Nombre científico: Ruta

graveolens

Familia: Rutaceae

Nombre común: Ruda

Nombre maya: $k^{\prime} a k^{\prime} a s$ ich
Uso

Medicinal: En las dolencias respiratorias causadas por procesos infecciosos, como catarro, resfriado, bronquitis, etc., elimina la tos. Desinflama los bronquios y ayuda a eliminar los gérmenes (se hierve y se toman cinco gotas de esencia al día). El líquido se pone en el oído para sanar el dolor. Las infusiones, bien calientes, de las flores de esta planta ayudan a reducir la fiebre: una cucharadita de flores secas por taza de agua, se deja reposar media hora y se toma un par de tazas al día, es importante tomarlas calientes. Contiene más de 30 antioxidantes, entre ellos el timol (muy abundante también en el tomillo), el ácido rosmarínico o el carvacrol. Sus propiedades antioxidantes pueden ser muy útiles en el tratamiento de enfermedades como el sida o el cáncer; se hace una infusión de una cucharada de té por taza de agua y se toman tres tazas al día.

Alimento: Frecuente en la cocina mexicana, especialmente en los tamales de ciertas zonas del sur de México: los pescados o carnes se envuelven en sus hojas fragantes para cocinar exquisitos platillos, como el tapixte y el pilte del sur de Veracruz. Además de emplearse como condimento, en la medicina tradicional las hojas se usan como estomáquicas, analgésicas y estimulantes. En homeopatía se usa la tintura de la planta entera, y las diluciones se prescriben contra el asma, la bronquitis, la laringitis aguda o crónica y la disnea.

Medicinal: Se pone a hervir con agua y se toma como té, para evitar vómitos.

Medicinal: Para el dolor de cabeza, mal de ojo, amenorrea, espasmos gastrointestinales, parasitosis, várices y hemorroides. Por vía externa, para tratar el vitíligo y la leucodermia. Es un buen remedio para aliviar la reuma y algunos trastornos oftalmológicos. Se toma en infusión como excelente calmante nervioso. Muy efectiva contra la sarna, aplicada sobre las partes afectadas. 


\section{Cuadro 1. Especies encontradas en Chancah Veracruz, y principales usos por los pobladores \\ (continuación)}

ESPECIE

Nombre científico: Talicia olivaeformis

Familia: Sapindaceae Nombre común: Huaya Nombre maya: Waya

Nombre científico: Virgilia capensis

Familia: Papilionaceae

Nombre común: Virgilia

Nombre maya: $O x$
Uso

\begin{abstract}
Alimento: El fruto, de semilla redonda y lisa, posee una cubierta jugosa color anaranjado, rica en azúcares y con una pequeña cantidad de taninos, una substancia astringente rica en vitaminas, en particular de vitamina $\mathrm{C}$.
\end{abstract}

Ornamental: Se coloca en las entradas de las casas o en el solar (jardín trasero) de la casa.

Fuente: Elaboración propia con base en las entrevistas aplicadas.

\section{Discusión y conclusiones}

La investigación etnobotánica ha adquirido relevancia por la acelerada pérdida del conocimiento tradicional y la reducción de la disponibilidad de muchas especies útiles, consecuencia de la degradación de los bosques tropicales y de otros hábitats naturales (Norris, Scott y Morales, 1999; De Clerck y NegrerosCastillo, 2000; Rebollar-Domínguez et al., 2008; Gómez-Álvarez, 2012). Resulta urgente rescatar ese conocimiento y documentar la información sobre las especies útiles para desarrollar nuevos medicamentos y, al mismo tiempo, evaluar el grado de amenaza de las especies, a fin de diseñar estrategias de conservación y contribuir a la protección de la biodiversidad, así como a la preservación y la reafirmación cultural de las generaciones futuras de habitantes de Chancah Veracruz.

\section{Agradecimientos}

Los autores agradecen a don Celestino, al señor Antonio Chan Quiñónez, a la señora Guillermina Noh Guille y a todos los pobladores de Chancah Veracruz por sus aportaciones a este trabajo; al biólogo Héctor González por impulsar 
esta clase de estudios; al doctor Luis Manuel Mejía Ortiz, al doctor Eduardo Puente Pardo, al doctor Alejandro Espinoza Tenorio y a la maestra en Ciencias Yanet López Reyes por sus correcciones, observaciones y aportaciones al presente escrito.

\section{Fuentes consultadas}

Anderson, E.N. et al. (2005). Las plantas de los mayas: Etnobotánica en Quintana Roo, México. San Cristóbal de las Casas: Comisión Nacional para el Conocimiento y Uso de la Biodiversidad (Conabio)/El Colegio de la Frontera Sur (Ecosur).

Anta-Fonseca, S. y P. Pérez-Delgado (2006). Atlas de experiencias comunitarias en manejo sostenible de los recursos naturales en Quintana Roo. México: Secretaría de Medio Ambiente y Recursos Naturales (Semarnat).

Barrera, A. (1979). "La taxonomía botánica maya”. Anales de la Sociedad Mexicana de Historia de la Ciencia y de la Tecnología, 5, 21-34.

Barrera-Marín, A. (2012). "La etnobotánica”. Etnobiología, 10, Clásicos de la etnobiología en México (suplemento 1). A. Argueta-Villamar, E. Corona-M. y A. Moreno-Fuentes (comps.), 94-97.

Bautista-Zúñiga, F. et al. (2004). Técnicas de muestreo para manejadores de recursos naturales. Pachuca: Universidad Nacional Autónoma de México/Universidad Autónoma de Yucatán/Consejo Nacional de Ciencia y Tecnología/ Instituto Nacional de Ecología.

Bello-Baltazar, E. et al. (2002). "Transdisciplina y sustentabilidad maya”. Ciencia Ergo Sum, 3, 260-272.

Bonnier, G. (1990). Los nombres de las flores. Barcelona: Ediciones Omega.

Bonnier, G. y G. de Layens (1999). Claves para la determinación de plantas vasculares. Barcelona: Ediciones Omega.

Camus, A.P. y R. Bustamante-Araya (1998). Módulo de ciencias naturales. Crecimiento poblacional. Santiago de Chile: Programa de Mejoramiento de la Calidad y Equidad de la Educación (Programa mece/Educación Media)Ministerio de Educación. 
Canales, M., T. Hernández, J. Caballero, A. Romo de Vivar, A. Durán, R. Lira (2006). Análisis cuantitativo del conocimiento tradicional de las plantas medicinales en San Rafael, Coxcatlán, Valle de Tehuacán-Cuicatlán, Puebla, México. Acta Botánica Mexicana, 75, 21-43.

Chan-Quijano, J.G. y M. López-Mejía (2012). "Riqueza etnobotánica de los senderos interpretativos de la Universidad de Quintana Roo, Cozumel, México”, en VIII Congreso Mexicano de Etnobiología. Villahermosa: Universidad Juárez Autónoma de Tabasco/Asociación Etnobiológica Mexicana, abril de 2012.

Cruz, S. (2000). "Estructura y aprovechamiento de vegetación secundaria en XHazil Sur y Anexos, un ejido de la zona maya de Quintana Roo”. Tesis de licenciatura. Chetumal: Instituto Tecnológico de Chetumal.

De Clerck, F.A.J. y P. Negreros-Castillo (2000). "Plant Species of Traditional Mayan Homegardens of Mexico as Analogs for Multistrata Agroforests”. Agroforestry Systems, 48 (3), 303-317.

Galletti, H. (1999). "La selva maya en Quintana Roo (1983-1996): trece años de conservación y desarrollo comunal”, en R.B. Primack et al. (eds.). La selva maya: Conservación y desarrollo. México: Siglo xxi Editores, 52-73.

Gómez-Álvarez, R. (2012). "Plantas medicinales de una aldea del estado de Tabasco, México”. Revista Fitotecnia Mexicana, 35, 43-49.

Hernández-Álvarez, N.G., M.M. Ávila-Uribe y A. Patiño-Siciliano (2009). "Estudio etnobotánico de las plantas medicinales utilizadas en Villa de Luvianos, Luvianos, Estado de México”, en M.A. Vázquez-Dávila et al. (eds.). Cultura y biodiversidad, paradigmas axiales del siglo XXI. Memorias del VII Congreso Mexicano de Etnobiología y I Congreso Latinoamericano de Etnobiología. Pachuca: Universidad Autónoma del Estado de Hidalgo, 9.

Leonti, M., O. Sticher y M. Heinrich (2003). "Antiquity of Medicinal Plant Usage in Two Macro-Mayan Ethnic Groups (Mexico)”. Journal Ethnopharmacology, $88,119-124$.

Norris, R., J. Scott y L.O. Morales (1999). "Ecoturismo basado en la comunidad en la selva maya: problemas y potencial”, en. R.B. Primack et al. (eds.). La selva maya: Conservación y desarrollo. México: Siglo xxi Editores, 374-390. Ochoa-Gaona, S., I. Pérez-Hernández y N.C. Jiménez-Pérez (2008). Descripción de las especies de árboles más comunes de la sierra de Tenosique, Tabasco, México. San Cristóbal de las Casas: Ecosur. 
Ochoa-Gaona, S. et al. (1990). Descripción de las especies. Informe final del Proyecto Etnobotánica de las Especies Forestales de la IV Región. Managua: Universidad Nacional Agraria.

------ (2012). Flora leñosa útil de la sierra de Tenosique, Tabasco, México. Tapachula: Ecosur.

Olmsted, C., A. López-Ornat y R. Durán-García (1983). “Vegetación de Sian Ka’an. Reporte preliminar”, en Sian Ka'an. Estudios preliminares de una zona en Quintana Roo propuesta como reserva de la biosfera. México: Centro de Investigaciones de Quintana Roo/Secretaría de Desarrollo Urbano y Ecología, 47-94.

Pulido-Salas, M.T. y L. Serralta-Peraza (1993). Lista anotada de las plantas medicinales de uso actual en el estado de Quintana Roo, México. Chetumal: Centro de Investigaciones de Quintana Roo.

Ramos-Prado, J.M. y L. Porter-Bolland (2002). Manual de colecta, identificación, registro y certificación de fuentes de germoplasma de las especies de la vegetación secundaria potenciales de manejo de la Reserva Ecológica El Edén. Chetumal: Consejo Nacional de Ciencia y Tecnología/Comisión Nacional Forestal.

Rebollar-Domínguez, S. et al. (2008). "Huertos familiares, una experiencia en Chancah Veracruz, Quintana Roo”. Polibotánica, 25, 135-154.

Varela-Carlos, M.E. (ed.) (1998). Enciclopedia de Quintana Roo, t. 3. México: Verdehalago.

Villarreal-Quintanilla, J.A. (2002). Introducción a la botánica forestal. México: Universidad Autónoma Agraria Antonio Narro.

Zamora-Cornelio, L.F., S. Ochoa-Gaona, G. Vargas-Simón, J. Castellanos-Albores y B.H.J. de Jong (2010). Germinación de semillas y clave para la identificación de plántulas de seis especies arbóreas nativas de humedales del sureste de México. Revista Biología Tropical, 58, 717-732. 